\title{
Research of anti-GAD and anti-IA2 autoantibodies by ELISA test in a series of Moroccan pediatric patients with diabetes type 1
}

\author{
O Belhiba ${ }^{1,2}$, Z Aadam ${ }^{3}$, L Jeddane, ${ }^{2,5}$, R Saile ${ }^{3}$, H Salih ALJ ${ }^{3}$, AA Bousfiha ${ }^{2,4}$, F Jennane ${ }^{1,2}$
}

1. Pediatric Endocrinology Unit, Hospital d'Enfant Abderrahim Harouchi Chu Ibn Rochd, Casablanca, Morocco.

2. Laboratory of Clinical Immunology, Allergy, and Inflammation LICIA, Faculty of Medicine and Pharmacy Hassan II University-Casablanca, Morocco.

3. Biology Laboratory and Health/Immune and Metabolic Pathology Team-Faculty of Sciences Ben M'Sik. Hassan II University-Casablanca, Morocco.

4. Clinical Immunology Unit, Infectious Department, Hospital d'Enfant Abderrahim Harouchi, CHU Ibn Rochd, Morocco.

5. National Reference Laboratory, Mohamed VI University of Health Sciences (UM6SS), Casablanca, Morocco.

\begin{abstract}
Introduction: Type I diabetes (T1D) is an autoimmune disease with a prediabetic, asymptomatic period characterized by the selective destruction of insulin-producing $\beta$ cells. During the pre-clinical phase, various auto-antibodies are generated against several beta cell antigens such as anti glutamate acid decarboxylase (Anti-GAD), anti tyrosine phosphatase (Anti-IA2). Today, the coupled detection of Anti-IA2 with that of Anti-GAD proves its great importance in the diagnosis and prediction of type 1 diabetes. The combined positivity for both antibodies has a specificity and a positive predictive value of $100 \%$. Objectives: In this work, we evaluate the diagnostic value of anti-GAD and anti-IA2 antibodies in a series based on 78 Moroccan subjects initially under 16, suspected T1D.

Results and Discussion: Our series consists mainly of $74 \%$ of newly diagnosed patients for T1D and $26 \%$ of confirmed diagnostic patients, of whom $52 \%$ are females. The mean age of diagnosis is $7 \pm 4$ years, the mean of HbA1c at the time of diagnosis is $11.63 \pm 2.16 \%$, and the percentage of family history in our series is $69 \%$. The proportion of positive results for anti-IA2 antibodies and anti-GAD antibodies are, respectively, $76.92 \%$ and $62.82 \%$, and $52.56 \%$ of patients are positive for both auto-antibodies. This study confirms that anti-GAD and anti-IA2 auto-antibodies assays can detect patients early and the autoantibodies can persist several years after diagnosis of type 1 diabetes.

Conclusion: This study confirmed the diagnosis and classification of T1D (type 1A) in 87.18\% of patients, and we reported that the prevalence of anti-GAD and anti-IA2 is higher in girls than in boys.

Keywords: Type 1 diabetes, autoimmunity, autoantibodies, anti-GAD, anti-IA2, ELISA, classification.

DOI: https://dx.doi.org/10.4314/ahs.v20i3.40

Cite as: Belhiba O, Aadam Z, Jeddane L, Saile R, Salih ALJ H, Bousfiba AA, et al. Research of anti-GAD and anti-IA2 autoantibodies by ELIS A test in a series of Moroccan pediatric patients with diabetes type 1. Afri Health Sci. 2020;20(3): 1337-1343. bttps://dx.doi. org/10.4314/abs.v20i3.40
\end{abstract}

\section{Introduction}

Type 1 (T1D) or insulin-dependent diabetes mellitus (IDDM) is an autoimmune disease, with the majority

\section{Corresponding author:}

Belhiba Ouijdane,

Pediatric Endocrinology Unit, Hospital

d'Enfant Abderrahim Harouchi Chu Ibn Rochd,

Casablanca, Morocco. Faculty of Medicine

and Pharmacy Hassan II University, 19 Rue

Tarik Ibnou Ziad, 9154 Casablanca, Morocco.

Tel: (+212) 0681070988;

Email: ouijdane.belhiba@gmail.com of cases (type 1A) resulting from the selective destruction of insulin-producing $\beta$-cells in the pancreatic islet in subjects with increased susceptibility. This occurs at a variable rate and becomes clinically symptomatic when approximately $80-90 \%$ of pancreatic $\beta$-cells are destroyed.

T1D accompanied by the absence of immunological markers is classified as type $1 \mathrm{~B}$ diabetes (idiopathic) ${ }^{1}$. The clinical phase of the disease is preceded by an asymptomatic period of variable duration, reflecting the consequence of a well-sustained autoimmune process in which several autoantibodies are generated against several beta cell antigens, such as anti-glutamic decarboxylase (anti-GAD), anti-insulin (IAA), anti-ty- 
rosine phosphatase (anti-IA2), anti-Islet cell (ICA) and anti-transporter 8 zinc (anti-ZnT8) ${ }^{2}$.

At least one of these autoantibodies is present in $95 \%$ of the individuals who have DT1, after the detection of the hyperglycemia. These auto-antibodies can be effectively used for the prediction of type 1 diabetes, and they can serve as early markers of DT1, given their persistence in patients' sera for years prior to the development of diabetes type 1, at the time of diagnosis and even after diagnosis ${ }^{3,4}$.

Currently, the best method of early detection and diagnosis of DT1 is based on the use of combined tests of these autoantibodies. The measurement of anti-GAD with anti-IA2 detects autoimmunity with the same frequency (approximately 85\%) as the measurement of anti-islet cell antibodies (ICA), which suggests to replacing the ICA with anti-GAD and anti-IA2 testing as a diagnosis tool for islets auto-immunity in children ${ }^{5,6}$. It was demonstrated that the ICA, but not the anti-GAD, disappeared a few years after the diagnosis of diabetes. This suggests that ICA can be more closely related to the damage to beta cells than anti-GAD ${ }^{7}$.

Anti-GAD is present in $80 \%$ of newly diagnosed diabetic children. They are directed against a $65 \mathrm{kDa}$ protein called glutamate acid decarboxylase (GAD). Human GAD is an enzyme found in the brain and pancreas, it catalyzes the decarboxylation of glutamic acid to 7-aminobutyric acid (GABA) with the release of $\mathrm{CO} 2^{8}$.

In the pancreas, GABA exerts anti-diabetic effects by acting on both islet $\beta$-cells and the immune system. Furthermore, GABA suppresses insulitis and the systemic production of inflammatory cytokines ${ }^{9}$.

Anti-IA2 antibodies are circulating antibodies found in $78 \%$ of type 1 diabetics at the time of diagnosis. They are directed against peptide fragments of 37 to $40 \mathrm{kDa}$, which are obtained after trypsinization of Langerhans islet homogenates.

IA2 (insulinoma-associated protein-2) is an intracellular protein, widely expressed in the body, which has a negative regulatory role on the insulin-signaling pathway. It plays a crucial role in pancreatic $\beta$ cells by regulating cell proliferation and apoptosis ${ }^{10}$.

The detection of anti-IA2 coupled with that of anti-ICA and/or anti-GAD confer a predictive value of 75 to $100 \%$ over the next five years in at-risk populations ${ }^{7}$.

In Morocco, according to the Ministry of Health, there are more than 2 million diabetics, including 15,000 children who are being followed up for a T1D ${ }^{11}$. Unfortunately, no investigation of autoantibodies in T1D has been published. We thus envisage a primary study in Morocco on the research of the anti-GAD and anti-IA2 antibodies in the diagnostic exploration of a series of 78 Moroccan subjects suspected of having type 1 diabetes.

\section{Patients and methods \\ Patients}

Our study concerns 78 children aged from 1 to 16 years who were followed up for an evocative table of T1D at Abderrahim Harouchi Children's University Hospital in Casablanca, Pediatric Endocrinology Unit. This was done in comparison to a series of confirmed diagnostic patients for type 1 diabetes.

Diabetes was defined according to the criteria set by ISPAD in $2018^{12}$.

The patients were all hospitalized with a clinical symptomatology evocative of inaugural T1D: a cardinal syndrome (polyuria, polydipsia, etc.) with or without ketoacidosis or ketosis. Some children already known to have T1D were hospitalized for a glycemic imbalance (hypoglycemia or diabetic ketosis).

Our patients are 74\% newly diagnosed with T1D and $26 \%$ with confirmed diagnoses.

\section{Methods}

\section{Clinical examination}

We studied the demographic, clinical, and biological characteristics of all patients in our series by analyzing their files through the Excel program. A questionnaire was well established in this sense, including age, sex, family history, complications, etc.

\section{A dosage of Anti-GAD and Anti-IA2 antibodies}

Throughout one year, in the Pediatric Endocrinology Unit at the Hospital University of Abderrahim Harouchi of Casablanca, sera were obtained mostly during the initial hospitalization at the onset of the disease-on average within 10 days of diagnosis - for newly diagnosed patients, as well as for confirmed diagnosis patients for type 1 diabetes.

Samples of patients were analyzed by ELISA tests using anti-GAD and anti-IA2 commercial kits (EUROIMMUN, Germany).

These are a quantitative in vitro assay for the detection of human autoantibodies against glutamic acid decarboxylase (GAD) and protein tyrosine phosphatase (IA2), with $99 \%$ of specificity for the Elisa kits. 
The anti-GAD or anti-IA2 ELISA uses the ability of autoantibodies to act bivalently and bridge the antigen
(GAD or IA2) immobilized on the microplate and the biotinylated antigen in the liquid phase (Fig 1).

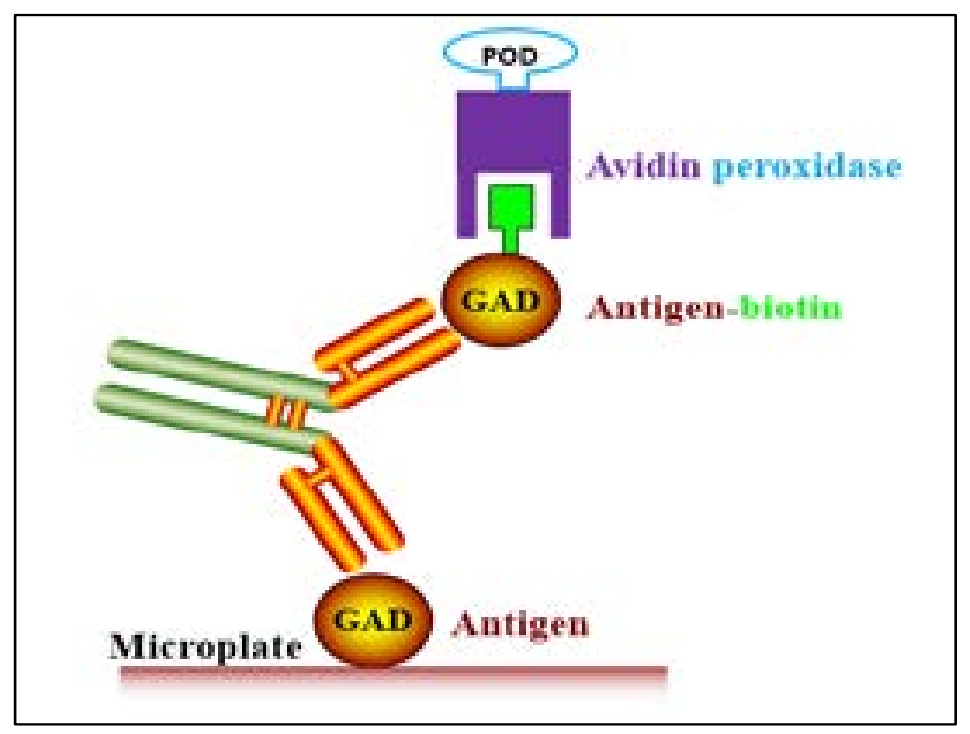

Fig 1 : Principle of the ELISA Anti-GAD / Anti-IA2.

In the first reaction step, patient samples are incubated in the wells. If samples are positive, specific antibodies bind to the IA2 or GAD.

Bound antibodies can act divalently and form a bridge between IA2 or GAD on reagent wells and biotin-labelled IA2/GAD, which is added in a second incubation step. To detect the bound biotin, a third incubation is carried out using enzyme-labelled avidin (enzyme conjugate), catalyzing a color reaction.

The intensity of the color formed is proportional to the concentration of antibodies against IA2.

The photometric measurement of the staining intensity is taken at $450 \mathrm{~nm}$ wavelength for low levels $<35 \mathrm{IU}$ $/ \mathrm{ml}$ and then at $405 \mathrm{~nm}$ for high concentrations $>35$
$\mathrm{IU} / \mathrm{ml}$.

According to the EUROIMMUN protocol, all the values of the results $<10 \mathrm{IU} / \mathrm{ml}$ are negative, and those $>10 \mathrm{IU} / \mathrm{ml}$ are positive.

\section{Results}

Demographic, Clinical, and Biological Characteristics

Our series consists of $74 \%$ of newly-diagnosed patients for T1D and $26 \%$ of confirmed diagnosis patients, with $52 \%$ of girls and $48 \%$ boys.

The mean age of the patients studied is $7 \pm 4$ years ranging from 1 to 16 years, and the median is 7 [4-11] (Table 1).

Table 1. Demographic, clinical and biological data of patients in our series.

\begin{tabular}{|c|c|}
\hline Age of patients (years) & $7 \pm 4$ \\
\hline Body weight (Kg) & $24 \pm 9,57$ \\
\hline BMI $\left(\mathrm{Kg} / \mathbf{m}^{2}\right)$ & $17,26 \pm 3,36$ \\
\hline Age at diagnosis (years) & $7 \pm 4,50$ \\
\hline Diabetes duration (months) & $9 \pm 20$ \\
\hline HbA1c (\%) & $11,63 \pm 2,16$ \\
\hline Fasting blood glucose (g / l) & $3,32 \pm 1,41$ \\
\hline
\end{tabular}

Mean \pm SD, BMI, body mass index; HbAlc, glycated hemoglobin.

The average $\mathrm{HbAlc}$ is $11.63 \pm 2.16 \%$, fasting blood glucose, reported for 39 patients $(48.13 \%)$, ranged from 1.1 to $8.4 \mathrm{~g} / 1$ and averaged $3.32 \pm 1.41 \mathrm{~g} / 1$. 
The mean age of diagnosis is $7 \pm 4.50$, and the median is 6 [4-9], with a diabetes duration ranging from a few days to 8 years.

The average HbA1c is $11.63 \pm 2.16 \%$; fasting blood glucose, reported for 39 patients (48.13\%), ranged from 1.1 to $8.4 \mathrm{~g} / \mathrm{l}$ and averaged $3.32 \pm 1.41 \mathrm{~g} / 1$.
Our results show that $69 \%$ of patients have a family history of diabetes, $44 \%$ of whom have that of a paternal origin, of which $19.5 \%$ have a type 1 diabetic father. The portion of ketoacidosis is $46 \%$, and the percentage of ketosis is $30 \%$ for newly diagnosed patients; there is $4 \%$ hypoglycemia, and 19\% imbalance for former patients (Fig 2).

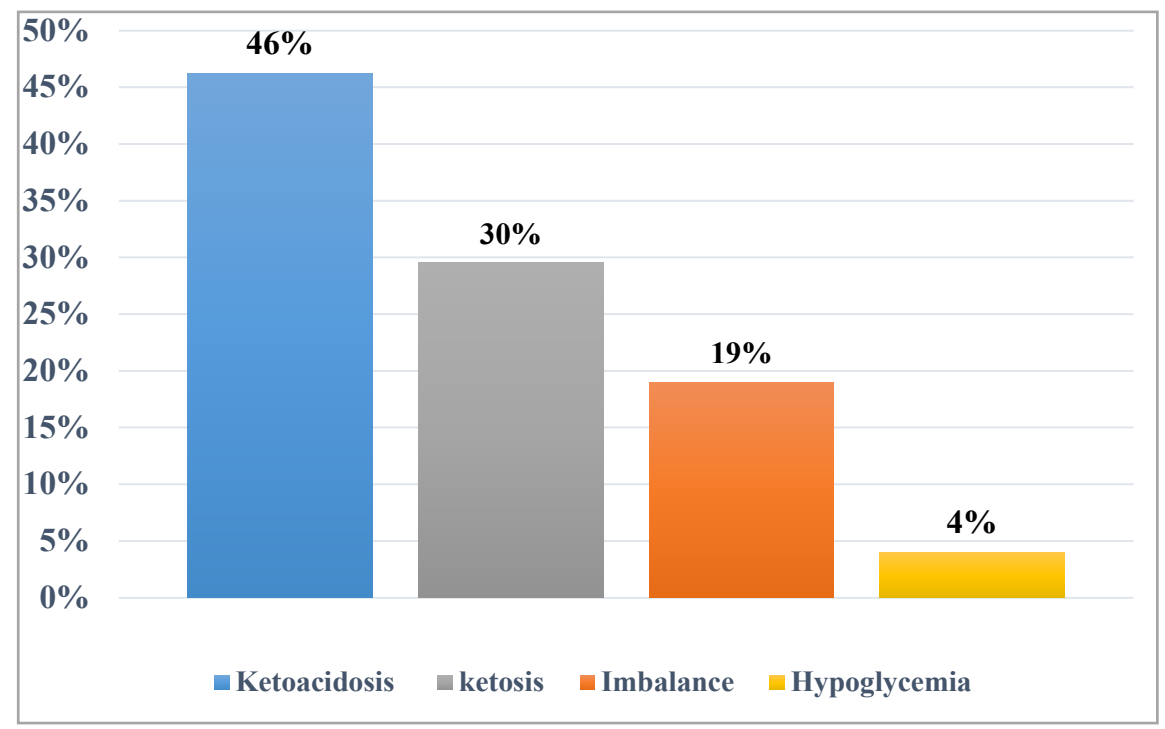

Fig 2 : Percentage of different reasons for hospitalization for type 1 diabetic patients (Ketoacidosis, Ketosis, Imbalance, Hypoglycemia).

Dosage of anti-GAD and anti-IA2 autoantibodies The proportion of positive results for anti-IA2 and anti-GAD antibodies are, respectively, $76.92 \%$ and $62.82 \%$;
$52.56 \%$ of patients are positive for both autoantibodies (anti-GAD, and anti-IA2), and only $12.82 \%$ of patients are negative for both autoantibodies (Fig 3).

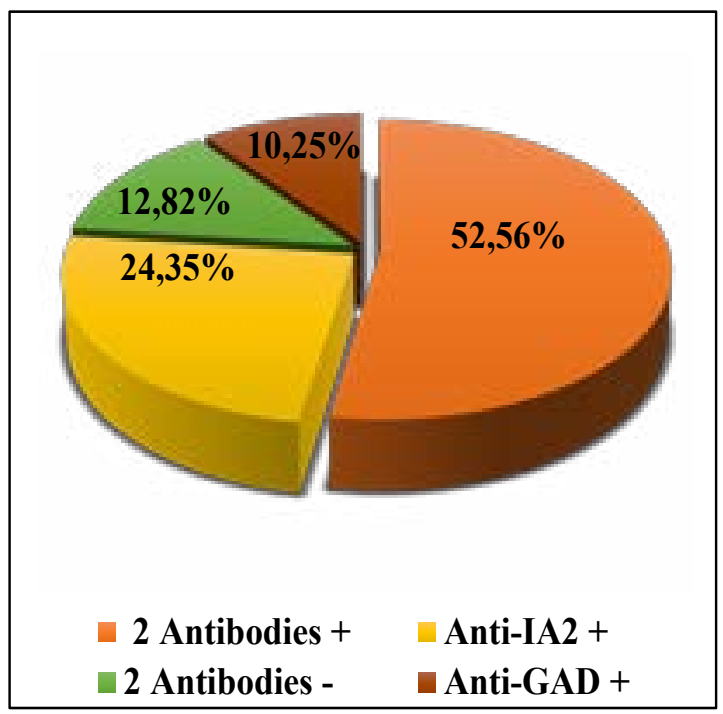

Fig 3 : Representation of the positivity of Anti-IA2 and Anti-GAD antibodies, the combination of 2 autoantibodies, and the percentage of 2 negative autoantibodies. 
The mean concentration of anti-IA2 is $139.41 \mathrm{IU} / \mathrm{ml}$, and the mean concentration of anti-GAD is $80.60 \mathrm{IU} /$ $\mathrm{ml}$; the median of the anti-GAD antibodies is $34.13 \mathrm{IU}$ $/ \mathrm{ml}$, and for antibodies against IA2, it is $19.62 \mathrm{IU} / \mathrm{ml}$. According to the results obtained, $87.18 \%$ of the patients in our series have type $1 \mathrm{~A}$ diabetes with one of the positive antibodies ${ }^{1}$, whereas $12.82 \%$ of the patients have both negative antibodies.

Among the $69 \%$ of patients with a family history of diabetes (53 patients), 61\% are positive for both auto-antibodies; $77 \%$ positive for anti-IA2, 61\% for anti-GAD For the 24 patients without a familial history of diabetes, $62.5 \%$ of them were positive for both autoantibodies, with $80 \%$ anti-IA2 positive and $66.7 \%$ anti-GAD positive.
We reported that $71 \%$ of girls are positive for the two antibodies as opposed to $54 \%$ of boys who are positive. We undertook to evaluate the evolution of the concentrations of these antibodies according to the duration of T1D ranging from a few days for newly diagnosed patients to 8 years of T1D for the oldest patients of our series.

We noted that $60 \%$ of former diabetic patients up to 8 years of T1D have positive results for both antibodies.

\section{Discussion}

The mean age at diagnosis in our series is $7 \pm 4.5$ years; this is close to that reported in other international series, such as Tunisia, with 8.16 years 13 , and Korea at 8.9 \pm 4.0 (14) (Table 2).

Table 2. Comparative table of the different demographic, clinical and biological characteristics of our series compared to other series.

\begin{tabular}{ccccc}
\hline & Our Series & $\begin{array}{c}\text { Tunisian Series } \\
\text { (2009) (14) }\end{array}$ & $\begin{array}{c}\text { Korean Series } \\
\text { (2017) (23) }\end{array}$ & $\begin{array}{c}\text { Chinese Series } \\
\text { (2016) (13) }\end{array}$ \\
\hline Age at diagnosis (years) & $7 \pm 4,50$ & 8,16 & $8,9 \pm 4,0$ & \\
Hba1c (\%) & $11,63 \pm 2,16$ & & $12.35 \%$ & $11,5 \pm 3$ \\
Family history (\%) & $69 \%$ & $21 \%$ pour le & $40 \%$ & $9,8 \%$ \\
& & T1D & & \\
Ketoacidosis (\%) & $46 \%$ & $23 \%$ & $49 \%$ & $44,6 \%$ \\
\hline
\end{tabular}

Mean \pm SD

HbA1C, glycated hemoglobin; T1D, type 1 diabetes.

The mean glycated hemoglobin in our series11, 63 is very close to that of the Chinese series $11.5 \pm 3$ and less than the average reported in the Korean series, which was $12.35 \%$.

We reported that $69 \%$ of our patients have a family history of diabetes, which can promote the increase of the risk factor to develop a T1D, and it may be due to a hereditary factor that has been transmitted from parents to their children.

Anti-IA2 is an antibody associated with the genotype DR4 and HLA class II of high-risk alleles (DRB1 * 0401 DQA1 * 0301 DQB1 * 0302 and DRB1 * 0402 DQA1 $* 0301 \mathrm{DQB} 1 * 0302)^{15}$.

This study shows that $76.92 \%$ of our patients are positive for anti-IA2, which may be due to the high genetic predisposition of the Moroccan population to T1D since our patients come from 15 different cities of Mo$\operatorname{rocco}^{16,17}$.

The early forms of T1D are marked by a high prevalence of high-risk class II HLA alleles (DRB1 * 03 and $* 04)$ and antibodies specifically directed against tyrosine phosphatase IA-2 and zinc transporter ZnT8 ${ }^{18}$. We can, therefore, conclude that $76.92 \%$ of our patients have an early form of T1D.

$12.82 \%$ of our newly diagnosed patients have both negative antibodies, may be either with type 1B diabetes or type 2 diabetes, but for the only former diabetic patient (known for 3 years) who is negative for both antibodies, this may be explained by the total absence of antigens due to the destruction of Langerhans beta cells, resulting in the negativation of autoimmune autoantibodies ${ }^{19}$. 
Humoral autoimmunity against the pancreatic islet, regarding of the prevalence of autoantibodies against GAD65 and IA2, is more common in diabetic women than in men $^{18}$.

The increased frequency of anti-GAD in women is in agreement with published data- $71 \%$ of girls are positive for the two antibodies as opposed to $54 \%$ of boys. In addition, the difference in the frequency of anti-GAD between women and men is more evident in the $11-20$ age group ${ }^{18}$, suggesting a possible involvement of female hormones in autoimmune processes.

In agreement with the previous reports, a higher prevalence of anti-IA2 is noted in the female diabetic group $(80.5 \%)$ compared to the male diabetic group $(73 \%)$. The anti-IA2 is the first marker to appear among children followed from birth to the development of diabetes ${ }^{20}$.

Half of all IA2-positive children remain 12 years after diagnosis, and anti-GAD can persist in the sera of the patients 8 years after the diagnosis 4 .

We noted that $60 \%$ of former diabetic patients up to 8 years of T1D have positive results for both antibodies. Therefore, both anti-GAD and anti-IA2 antibodies can persist and be detected several years after the diagnosis of type 1 diabetes.

We agree with the findings of Quirine $T$ et al. in their recent study published in 2016 on 990 diabetic patients, which confirms the persistence of anti-GAD several years after the diagnosis of $\mathrm{T}^{2} \mathrm{D}^{21}$.

\section{Conclusion}

This study confirmed the diagnosis and the classification of T1D (type 1A) in $87.18 \%$ of our patients, and in agreement with published data, we reported that the prevalence of Anti-GAD and Anti-IA2 is higher in girls than in boys.

The percentage of family history in our series is very important compared to neighboring countries (Algeria and Tunisia) ${ }^{22,14}$; we probably have a particular genetic profile that is favorable, with this hypothesis of genetic predisposition.

This genetic predisposition needs to be confirmed by HLA typing, looking for other genes, and an increase in sampling is required.

This study confirms that Anti-GAD and Anti-IA2 can persist for several years after the diagnosis of T1D.

\section{Conflict of interest}

The authors declare no financial or commercial conflict of interest.

\section{Ethics statement}

The Ethics Committee of Faculty of Medicine and Pharmacy Hassan II University approved the study with the Helsinki declaration of 2008, concerning human and animal rights, and written consent was obtained from the children's parents or guardians after describing the study in detail.

\section{Acknowledgements}

We are greatly indebted to Dr. Ibrahim khalil Ahmadaye for a statistical help, Dr. Leila jeddane and Dr. Zahra Aadam for technical help and technical advice, the Pediatric Endocrinology Unit, Hopital d'Enfant Abderrahim Harouchi Chu Ibn Rochd (Professor Farida Jennane), the Laboratory of Clinical Immunology, Allergy and Inflammation (Professor Ahmed Aziz Bousfiha), the Biology Laboratory and Health/ Immune and Metabolic Pathology Team (Professor Hanane Salih Alj, Professor Rachid Saile), the Faculty of Sciences Ben M'Sik. Hassan II University, and the Faculty of Medicine and Pharmacy Hassan II University.

\section{References}

1. Craig ME, Jefferies C, Dabelea D, Balde N, Seth A, Donaghue KC. Definition, epidemiology, and classification of diabetes in children and adolescents: Definition, epidemiology, and classification of diabetes. Pediatric Diabetes. 2014; 15(S20): 417.

2. Oikawa Y, Tanaka H, Uchida J, Atsumi Y, Osawa M, Katsuki T, et al. Slowly progressive insulin-dependent (type 1) diabetes positive for anti-GAD antibody ELISA test may be strongly associated with a future insulin-dependent state. Endocrine Journal. 2017; 64 (2): 163 70.

3. Kikkas I, Mallone R, Larger E, Volland H, Morel N. A Rapid Lateral Flow Immunoassay for the Detection of Tyrosine Phosphatase-Like Protein IA-2 Autoantibodies in Human Serum. Sechi LA, éditeur. PLoS One. 2014; 9 (7): e103088.

4. Durand G, Beaudeux J-L. Biochimie médicale: Marqueurs actuels et perspectives. Lavoisier, 2011. 643 p.

5. Borg H, Fernlund P. Protein tyrosine phosphatase-like protein IA2- antibodies plus glutamic acid decarboxylase 65 antibodies (GADA) indicates autoimmunity as frequently as islet cell antibodies assay in children with recently diagnosed diabetes mellitus. Clinical Chemistry. 1997; (12): 6.

6. Seissler J, Morgenthaler NG, Achenbach P, Lampeter EF, Glawe D, Payton M, et al. Combined screening for autoantibodies to IA-2 and antibodies to glutamic acid 
decarboxylase in first degree relatives of patients with IDDM. Diabetologia. 1996; 39(11): 13516.

7. Nonnenmacher L. Informations Réactifs : Insuline, Peptide C, anticorps anti-ICA, anti-GAD65, anti-IA2 et anti-insuline. Immuno-analyse \& Biologie Spécialisée. 2002; 17(3): 18598.

8. Rharbaoui F, Bouanani M, B Pau CG. L'isoforme de $65 \mathrm{kDa}$ de l'acide glutamique décarboxylase (GAD65). Immuno-analyse Biol Spécialisée. 1998; 13: 219-221.

9. Soltani N, Qiu H, Aleksic M, Glinka Y, Zhao F, Liu $\mathrm{R}$, et al. GABA exerts protective and regenerative effects on islet beta cells and reverses diabetes. Proceedings of the National Academy of Sciences. 2011; 108(28): 11692 7.

10. Fernandez-Ruiz R, Vieira E, Garcia-Roves PM, Gomis R. Protein Tyrosine Phosphatase-1B Modulates Pancreatic $\beta$-cell Mass. Kay TW, éditeur. PLoS One. 2014; 9(2): e90344.

11. Louardi expose les chiffres effrayants du diabète au Maroc. Nouvelle Tribune. 2016; Available from: «http://lnt.ma/louardi-expose-les-chiffres-effrayantsdu-diabete-au-maroc».

12. Mayer-Davis EJ, Kahkoska AR, Jefferies C, Dabelea D, Balde N, Gong CX, et al. ISPAD Clinical Practice Consensus Guidelines 2018: Definition, epidemiology, and classification of diabetes in children and adolescents. Pediatric Diabetes. 2018; 19: 719.

13. ten Kate Q, Aanstoot HJ, Birnie E, Veeze H, Mul D. GADA persistence and diabetes classification. The Lancet Diabetes \& Endocrinology. 2016; 4(7): 5634.

14. Hachicha M, Aloulou H, Bouraoui A, Ben Ameur S, Elloumi S, Chabchoub I, et al. P199 Les aspects épidémiologiques du diabète de type 1 : expérience du service de pédiatrie de Sfax (Tunisie). Diabetes \& Metabolism. 2009; 35: A75.

15. Yang J, Zhou M-C, Feng K, Wang O, Zhang H-B, Li W, et al. Clinical Characteristics of 261 Cases of Hos- pitalized Patients with Type 1 Diabetes Mellitus. Chinese Medical Sciences Journal. 2016; 31 (2): 6975.

16. Izaabel H, Garchon H-J, Beaurain G, Biga M, Akhayat O, Bach J-F, et al. Distribution of HLA class II alleles and haplotypes in insulin-dependent moroccan diabetics. Human Immunology. 1996; 49 (2): 13743.

17. Drissi Bourhanbour A, Benseffaj N, Ouadghiri S, Razine R, Touzani A, Belafraj A, et al. Family-based association study of HLA class II with type 1 diabetes in Moroccans. Pathologie Biologie. 2015; 63 (2): 804.

18. Arif S, Leete P, Nguyen V, Marks K, Nor NM, Estorninho $\mathrm{M}$, et al. Blood and Islet Phenotypes Indicate Immunological Heterogeneity in Type 1 Diabetes. Diabetes. 2014; 63 (11): 383545.

19. Papadimitriou DT, Marakaki C, Fretzayas A, Nicolaidou P, Papadimitriou A. Negativation of type 1 diabetes-associated autoantibodies to glutamic acid decarboxylase and insulin in children treated with oral calcitriol: Calcitriol and type 1 diabetes. Journal of Diabetes. 2013; 5 (3): 3448.

20. Bilbao JR, Rica I, Vázquez JA, Busturia MA, Castaño L. Influence of Sex and Age at Onset on Autoantibodies against Insulin, GAD65 and IA2 in Recent Onset Type 1 Diabetic Patients. Hormone Research in Paediatrics. 2000; 54 (4): 1815.

21. Achenbach P, Bonifacio E, Koczwara K, Ziegler A-G. Natural History of Type 1 Diabetes. 2005; 54: 7.

22. Bensenouci A, Achir M, Boukari R, Bouderda Z, Lacete F, Kaddache C, et al. La prise en charge du diabète de type 1 chez l'enfant en Algérie (DiabCare Pédiatrique). Médecine des Maladies Métaboliques. 2014; 8 (6): 64651.

23. Lee HJ, Yu HW, Jung HW, Lee YA, Kim JH, Chung HR, et al. Factors Associated with the Presence and Severity of Diabetic Ketoacidosis at Diagnosis of Type 1 Diabetes in Korean Children and Adolescents. Journal of Korean Medical Science. 2017; 32 (2): 303. 